\title{
Real Time Seismic Monitoring System for Earthquake Using GPS Technology
}

\author{
Neha Handa ${ }^{1}$ \\ ${ }^{I}$ Department of Electrical \& Electronics Engineering, GBTU University, India
}

\begin{abstract}
As part of earthquake process, earth's surface is being deformed as earthquake faults accumulate strain \& slip or slowly creep over time. We use GPS to monitor this movement by measuring precise position (within $5 \mathrm{~mm}$ or less) of stations near active faults relative to each other. In this paper, seismic monitoring is made through a real time analysis using GPS technology. This paper explains how GPS will be used to measure the movement of the earth, i.e., how far the earth actually moved \& in what direction, this may be in form of lateral movement or vertical displacement. Here GPS enables direct fault motion measurement in earthquake using one or more GPS's three basic components, i.e. absolute location, relative movement \& time transfer. The technology here uses real time GPS information to measure vital stats on a quake. The technology use is referred as, "Real Time Earthquake Analysis For Disaster, (READI)". Because of its accuracy \& precision, the technology has played a vital role in sensing the area précised to $5 \mathrm{~mm}$. We here are using the GPS data automatically to calculate the location, magnitude \& other details about the earthquake fault. GPS receiver is vital component in are paper because it is stationed to receive the satellite's signal, so that movement could be measured with pinpoint accuracy. It will also measure the high frequency carrier wave used to send the code. Its accuracy is lot higher, providing more precise positional information.
\end{abstract}

Keywords: GPS receiver, GPS technology, READI, Seismic monitoring, Sierra monitoring system

\section{Introduction}

The present technology in seismic instrumentation and telecommunications permits the implementation of a computerized system for earthquake early warning. Such a system is capable of providing from a few seconds to a few tens of seconds of warning before the arrival of strong ground shaking caused by a large earthquake. This timely information may be used (1) to minimize property damage and the loss of lives in metropolitan areas, and (2) for real-time loss estimation to aid emergency response and recovery. The Global Positioning System (GPS) is a space-based satellite navigation system that provides location and time information in all weather conditions, anywhere on or near the Earth where there is an unobstructed line of sight to four or more GPS satellites. The system provides critical capabilities to military, civil and commercial users around the world. It is maintained by the United States government and is freely accessible to anyone with a GPS receiver. Advances in technology and new demands on the existing system have now led to efforts to modernize the GPS system. The current GPS consists of three major segments. These are the space segment (SS), a control segment (CS), and a user segment (US). GPS satellites broadcast signals from space, and each GPS receiver uses these signals to calculate its three-dimensional location (latitude, longitude, and altitude) and the current time.

\subsection{GPS a precise instrument}

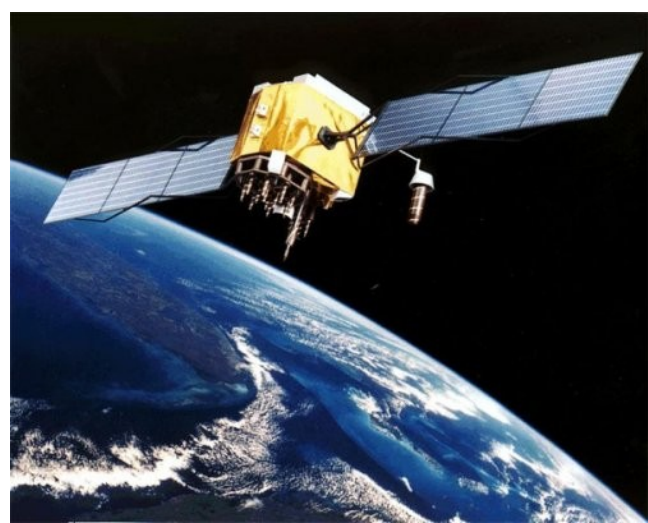

Fig1: GPS in earthquake study

When driving from point A to point B you may not require accuracy to the centimeter, normally within a meter or two is fine. But GPS is an incredibly precise tool that can measure down to the millimeter, and that's 
why it's proving useful to geologists when tracking earthquakes around the world. However you won't find your average garden-variety GPS device used in these situations. Because movement needs to be measured with pinpoint accuracy, a sophisticated GPS receiver is stationed to receive the satellite's signal.

The GPS receiver not only uses and stores the satellites code (which provides location information) it also measures the carrier wave used to send the code. Since this carrier wave is a much higher frequency, its accuracy is a lot higher, providing more precise positional information.

In fact, the information recorded by these GPS receivers, along with some powerful computer software, allows scientists to measure the movement of two points anywhere on the Earth's surface to within $5 \mathrm{~mm}$ !

Precise monitoring is mainly required to monitor the earthquake. Therefore we have considered GPS system much more effective in comparison to seismometer.

By Relative Kinematics Positioning (RKP) an alternative for a precise GPS-based positioning system we can precisely monitor the range of signal. In this approach, determination of range signal can be resolved to a precision of less than 10 centimeters $(3.9 \mathrm{in})$. This is done by resolving the number of cycles that the signal is transmitted and received by the receiver by using a combination of differential GPS (DGPS) correction data, transmitting GPS signal phase information and ambiguity resolution techniques via statistical tests-possibly with processing in real-time (real-time kinematics positioning, RTK).

\subsection{Basic concept of GPS}

GPS receivers record the earth's movements during an earthquake. A GPS receiver calculates its position by precisely timing the signals sent by GPS satellites high above the Earth. Each satellite continually transmits messages that include

- the time the message was transmitted

- satellite position at time of message transmission

The receiver uses the messages it receives to determine the transit time of each message and computes the distance to each satellite using the speed of light. Each of these distances and satellites' locations define a sphere. The receiver is on the surface of each of these spheres when the distances and the satellites' locations are correct. These distances and satellites' locations are used to compute the location of the receiver using the navigation equations. This location is then displayed, perhaps with a moving map display or latitude and longitude; elevation information may be included. Many GPS units show derived information such as direction and speed, calculated from position changes. In GPS operation we generally use four or more satellites to have precise and accurate result and thing to be noted is that none of these satellites intersect each other. As a result we could easily make out that the result that we will conclude by solving the navigation equations to find an intersection; this solution gives us the position of the receiver along with accurate time, which in returns reduces the need of having large expensive clocks. Because of this advance feature we get the accurate timings to manage the disaster like earthquake.

\section{How is GPS used to measure earthquakes?}

In real-time monitoring, signals from seismic sensors in the field (either analog or digital) are telemetered to a central receiving station for processing. By real-time we mean that the results can be obtained within seconds or tens of seconds. In practice, there are several obstacles to achieving a quicker response. Large earthquakes normally occur at depth of a few tens of kilometers or deeper. It takes several seconds for seismic waves to reach the earth's surface where the seismic sensors are located, and several tens more seconds before sufficient numbers of sensors detect the seismic waves. In the experimental earthquake early warning system in Hualien, Taiwan, a 10-second or less response time has been achieved for earthquakes 5 occurring inside or near the dense array with sensor spacing of about $2 \mathrm{~km}$. However, such dense deployment of sensors is not economical to cover a large area in real practice. In essence, a real-time seismic monitoring system consists of: (1) sensors deployed in the field, (2) telemetry, (3) a central receiving station where real-time data acquisition and processing are performed, and (4) if an potential damaging earthquake has been detected, then results are communicated via one or more communication channels to users. For an earthquake early-warning system based on real-time seismic monitoring, we must achieve a response time that users can take actions before strong shaking arrives. Tracking earthquake movements using GPS is just one more use for the amazing technology that has become ubiquitous in the $21^{\text {st }}$ century. Recently NASA is using GPS technology to find and respond to the earthquakes. Generally, earthquake detection is done by seismological hardware on the ground, but according NASA - that data can be difficult to capture. NASA's answer to that problem is to use the GPS satellites in orbit along with GPS hardware on the ground in a new network called the Real-Time Earthquake Analysis for Disaster Mitigation Network. The system is called READI. The READI Mitigation Network will use real-time GPS measurements to calculate the characteristics of large earthquakes to aid early warning and disaster relief efforts. These systems allow earthquake parameters to be broadcasted to users in a few minutes after the earthquake occurred. 
"Recent advances in seismic sensor technology, data acquisition systems, digital communications, and computer hardware and software make it possible to build reliable real-time earthquake information systems.

Such systems provide a means for modern urban regions to cope effectively with the aftermath of major earthquakes and, in some cases, they may even provide warning, seconds before the arrival of seismic waves. In the long term these systems also provide basic data for mitigation strategies such as improved building codes."

Before you leap up to point out that this article is completely wrong because GPS is not used to measure earthquakes, let's quickly clarify something. The GPS receiver is not used to measure the strength of the quake. That's the job of a seismograph, often the type seen is a Kinematics seismograph, the paper roll drum with a pointer charting any movement detected by sensors placed in a seismic station, normally situated under the ground. GPS is used to measure the movement of the earth, that is how far the earth actually moved and in what direction. This may be in the form of lateral (sideways) movement or vertical displacement.

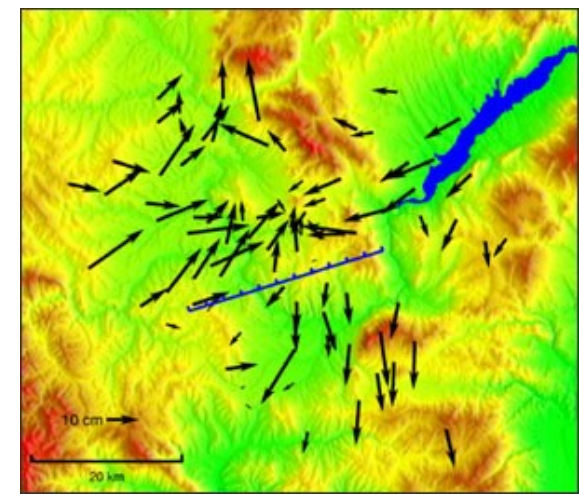

Fig.2: GPS receivers record the earth's movements during an earthquake

For example, looking at the image shown above you'll notice arrows. These arrows are generated by information recorded by several different GPS receivers. It shows the ground on either side of the fault being moved apart during the earthquake. This type of fault is called a normal fault and is common in some places where the crust is being stretched. Geologists can use this information to monitor the earth's movement, predicting future changes, and using it to help building planners and surveyors, as well as understanding better how earthquakes affect the land around them.

The network uses real-time GPS measurements, when a large earthquake is detected, GPS data are used to automatically calculate its vital characteristics including location, magnitude and details about the fault rupture.

"With the READI network we are enabling continued development of real-time GPS technologies to advance early warning disaster systems". "This prototype system is a significant step towards realizing the goal of providing effective and real time monitoring and risk mitigation of earthquake.

Accurate and rapid identification of earthquakes of magnitude 6.0 to 7.0 and stronger is critical for disaster response and mitigation efforts. Calculating the strength of a earthquake requires detailed knowledge of the associated ground movements. Acquiring this type of data for very large earthquakes is a challenge for traditional seismological instruments that measure ground shaking.

High-precision, second-by-second measurements of ground displacements using GPS have been shown to reduce the time needed to characterize large earthquakes and to increase the accuracy of subsequent tsunami predictions. After the capabilities of the network have been fully demonstrated, it is intended to be used by appropriate natural hazard monitoring agencies. USGS and the National Oceanic and Atmospheric Administration are responsible for detecting and issuing warnings on earthquakes and tsunamis, respectively.

"By using GPS to measure ground deformation from large earthquakes, we can reduce the time needed to locate and characterize the damage from large seismic events to several minutes".

$\mathrm{T}$ he READI network is a collaboration of many institutions including Scripps at the University of California in San Diego; Central Washington University in Ellensburg; the University of Nevada in Reno; California Institute of Technology/Jet Propulsion Laboratory (JPL) in Pasadena; UNAVCO in Boulder, CO; and the University of California at Berkeley.

NASA, NSF, USGS, and other federal, state, and local partners support the GPS stations in the network, including the Earth Scope Plate Boundary Observatory, the Pacific Northwest Geodetic Array, the Bay Area Regional Deformation Array and the California Real-Time Network. 


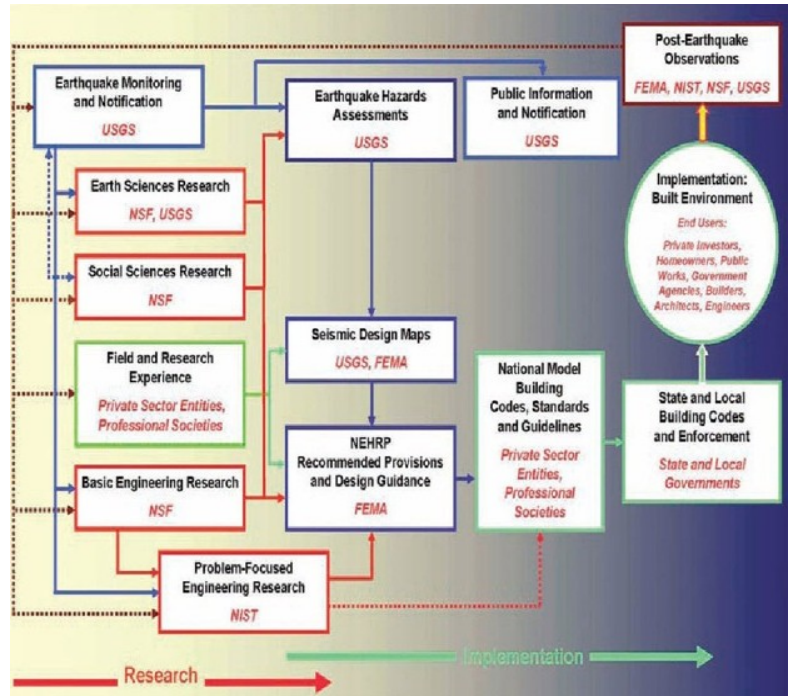

Fig3.: National hazard reduction program

"The relatively small investments in GPS-based natural hazards systems have revolutionized and allowed us to develop this prototype system with great potential benefits for the infrastructure and population in earthquake-prone states ". The READI network is the outgrowth of developing a global GPS signal receiving network to improve the accuracy and utility of GPS positioning information. This capability provides real-time, pinpoint positioning and timing helps to monitor the Earth.

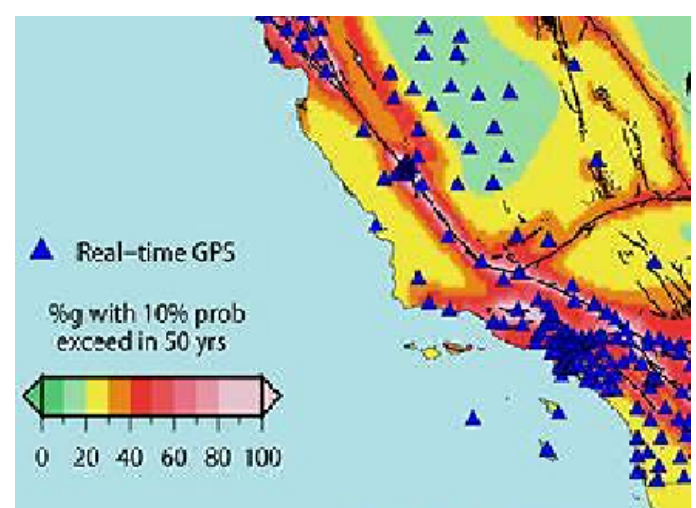

Fig 4: GPS station for Real- Time Analysis and disaster mitigation

"Conventional seismic networks have consistently struggled to rapidly identify the true size of great earthquakes during the last decade". But this GPS system technology has likely provided us with the accurate and rapid estimates of the location and amount of fault slip so that precise monitoring of quakes could be made easily. The GPS earthquake detection capability was first demonstrated by NASA-supported research on a major 2004 Sumatra quake conducted by Geoffrey Blewitt and colleagues at the University of Nevada in Reno.

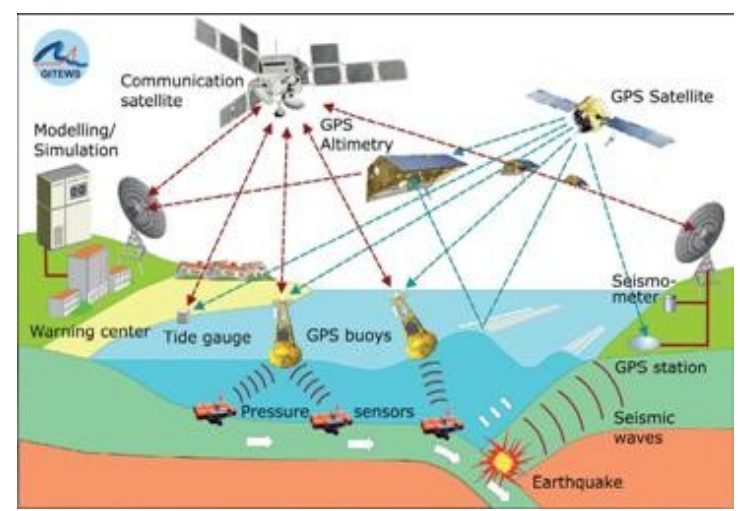

Fig 5: Schematic diagram representing the complete system of monitoring earthquake using GPS technology 


\section{Assessment of measurement accuracy of GPS}

The GPS technology is an emerging tool for measuring and monitoring both static and dynamic displacement responses of slender engineering structures to ambient loads. As a relatively new

Table I. Accuracy levels for different GPS positioning modes.

\begin{tabular}{|l|l|l|l|}
\hline Item & Static & Fast Static & RTK \\
\hline $\begin{array}{l}\text { Time required to calculate a } \\
\text { position }\end{array}$ & Several hours & $8-25 \mathrm{~min}$ & Approximately 15 s \\
\hline Positions measured within & $0.5-2 \mathrm{~cm}$ & $1-5 \mathrm{~cm}$ & $1-5 \mathrm{~cm}$ \\
\hline
\end{tabular}

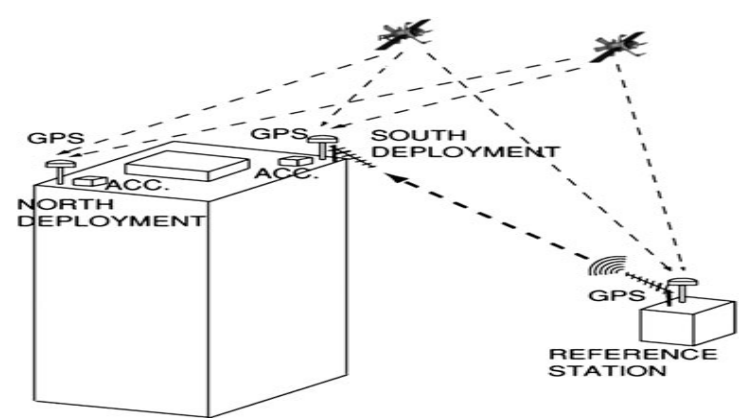

approach, the GPS performance must be thoroughly validated before its application in full scale. Many researchers have conducted feasibility trials to investigate the following problems (1) What is the range and level of accuracy of modal frequencies determined with the GPS? (2) Does this accuracy depend on the frequency of recorded oscillations? (3) Can variations in modal frequencies and multiple modal frequencies in a single displacement record be identified?

\section{Progress on monitoring the displacement of the high- rise structure caused by the ambient effects}

The GPS offers great potential for tall building health monitoring applications. Their significance to health monitoring applications stems from the following facts: (1) There is no intervisibility among stations, and the observation values are independent. (2) All-weather operations. The GPS signal receiver can work continuously at any place and any time, generally free of the impact of weather conditions. (3) High-precision positioning. Real-time positioning accuracy can be up to $10 \mathrm{~mm}$ for plane, $20 \mathrm{~mm}$ for height. (4) Short observation time. The output time delay of high-precision points is shorter than $0.05 \mathrm{~s}$. (5) The static and dynamic 3D coordinates can be determined simultaneously. (6) The results have no error accumulation and are zero drift. (7) Easy to operate. The GPS measurements have high automation; thus, it is easy to build the automated continuously operating system .From the aforementioned discussion, it would be correct to believe that the GPS is capable as a useful tool for (1) detecting tall building response in real time to extreme loading events such as windstorms with high velocities and earthquakes of average to high magnitudes, (2) estimating the permanent' displacements experienced by tall buildings once the shaking event has stopped, and (3) detecting long-term tall building deformation due to ground subsidence and temperature variation. The integration of the GPS with high-rise structures for the SHM has become an active research field. Although the benefits of GPS long-term structural monitoring are yet to be fully realized, several applications have been demonstrated to date. The rest of this section will review these demonstrations.

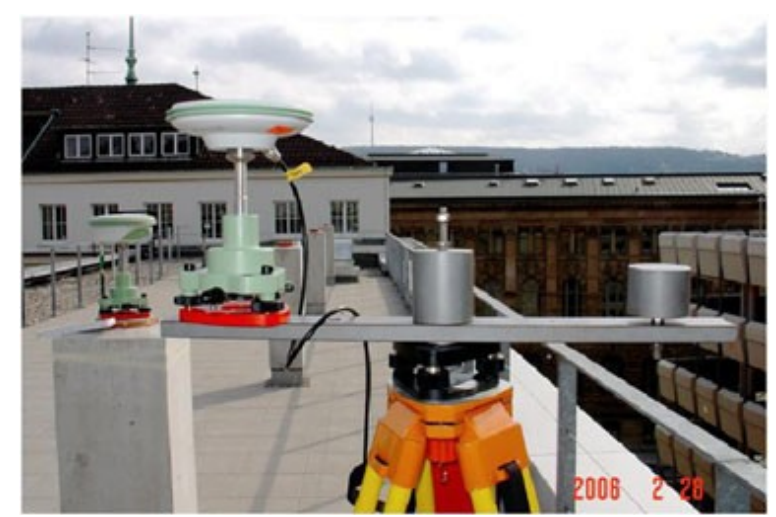

Figure 6. Rover Antenna mounted on a tripod provided with a rotating arm 


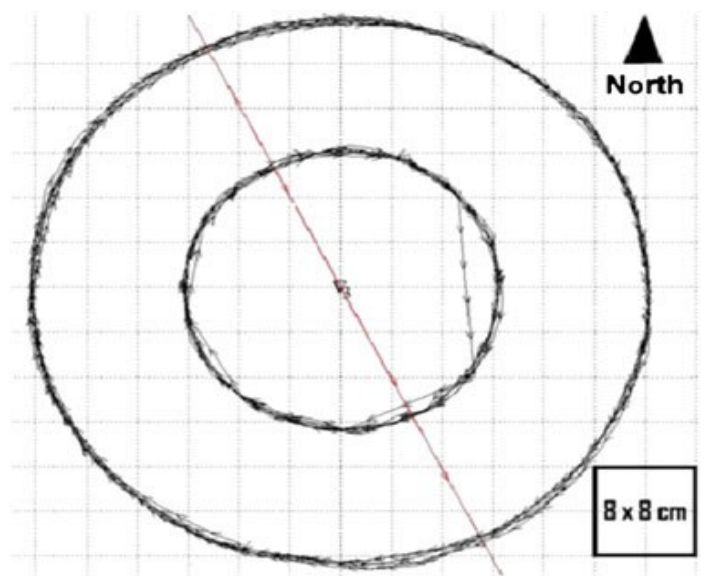

Figure 7. Result of the Rover Antenna's kinematic GPS tracing applied on two different radii

\section{Sierra seismic monitoring system}

Kinemetrics' Sierra provides rapid information for earthquake mitigation by monitoring seismic events from local, regional and national networks and arrays. Sierra is based on the Altus recorders to digitize and transmit data over full duplex telemetry links and a PC based data acquisition and on-line processing system. The system runs under Microsoft Windows to provide a user friendly environment, making system setup and data display quick and easy. Sierra offers the advantages of recording seismic events to the Altus recorder's removable PC memory card when the trigger criteria are met, providing a backup system for the network. The Altus recorder can also be connected to a triaxial force balanced accelerometer to provide information about ground motion.

\subsection{Sierra field station}

At remote sites, the Sierra field station consists of seismometers and/or accelerometers, an Altus recorder and communication interface. The Altus recorder converts analog signals from the transducers to digital format and time stamps the data for all channels using the GPS receiver. All of Kinemetrics' Altus recorders (Etna, K2 or Everest) can be installed at field stations. The communication interface transfers continuous data to the Sierra data centre using standard duplex serial interface over radio, telephone or satellite communication links

\subsection{Sierra data centre}

The Sierra data centre consists of the data concentrator, the real-time data acquisition and processing system, and the off line analysis system.

The data concentrator merges all incoming data streams and forward them to the real-time data acquisition and processing system. The real time acquisition and processing system records all the incoming signals and performs seismic event detection using Kinemetrics' Network Management System (NMS).

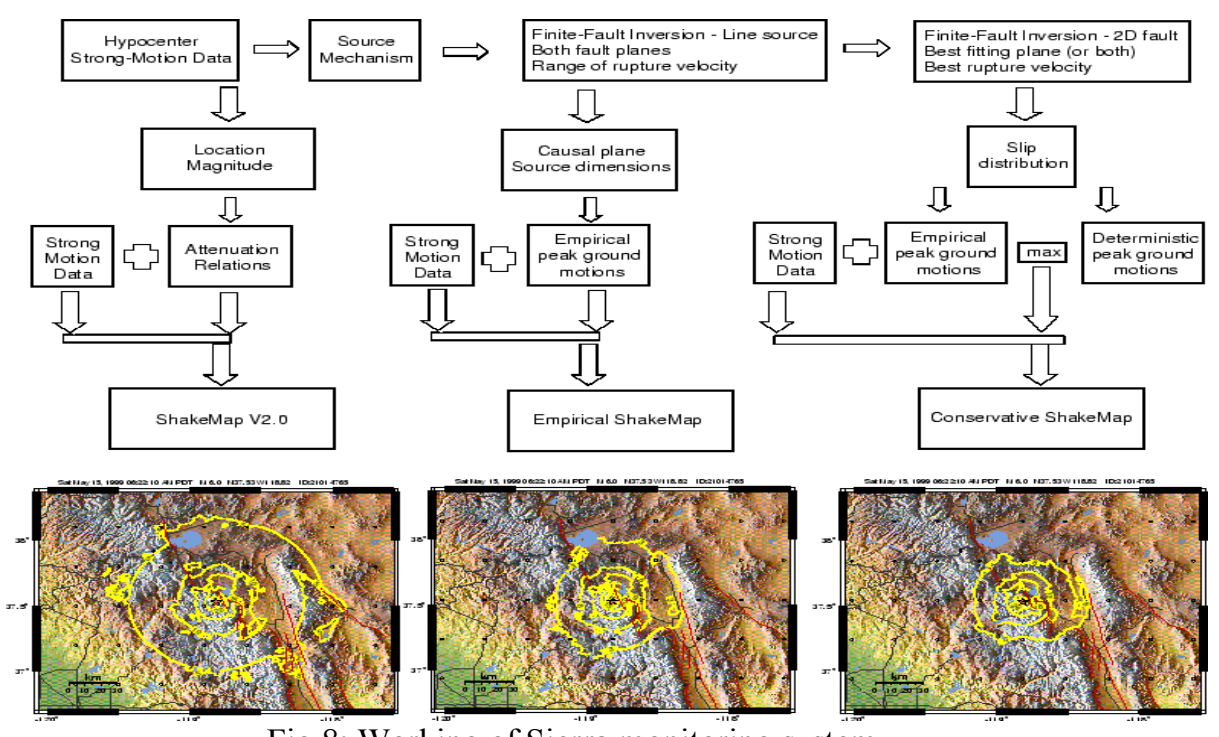

Fig.8: Working of Sierra monitoring system 
Hypocenter and magnitude processing is performed automatically after an event has been detected and the information is then graphically displayed on the map. The off-line computer incorporates the laspei and seisan seismic analysis software packages of the antelope seismic information system (ASIS). Seisan provides a complete set of utilities with a simple database for analyzing earthquake data. A large selection of tools is available for further processing from Siesan and laspei including signal spectral parameter determination, seismic moment, azimuth and epicenter plotting. ASIS uses relational database (RDBMS) formalism and the CSSv.3.0 schema for information organization. ASIS comes with complete set of tools for processing seismic data and runs on the Sun Microsystems' Solaris operating system on both SPARC and Intel architectures.

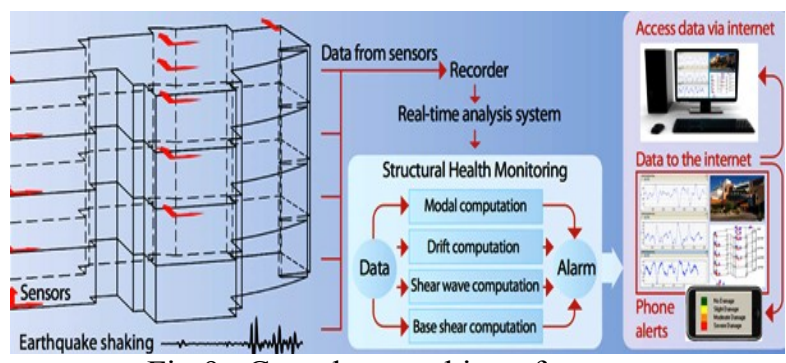

Fig 9: Complete working of system

\section{Navigation equations}

The receiver uses messages received from satellites to determine the satellite positions and time sent.

The $x, y$, and $z$ components of satellite position and the time sent are designated as $\left[x_{i}, y_{i}, z_{i}, t_{i}\right]$ where the subscript $i$ denotes the satellite and has the value $1,2, \ldots, n$, where $\mathrm{n} \geq 4$ When the time of message reception indicated by the on-board clock is $t_{r}$, the true reception time is $t_{r}+b$ where $b$ is receiver's clock bias (i.e., clock delay). The message's transit time is $\mathrm{t}_{\mathrm{r}}+\mathrm{b}-\mathrm{t}_{\mathrm{i}}$. Assuming the message traveled at the speed of light, $C$, the distance traveled is $\left(t_{r}+b-t_{i}\right) c$. Knowing the distance from receiver to satellite and the satellite's position implies that the receiver is on the surface of a sphere centered at the satellite's position with radius equal to this distance. Thus the receiver is at or near the intersection of the surfaces of the spheres if it receives signals from more than one satellite. In the ideal case of no errors, the receiver is at the intersection of the surfaces of the spheres.

The clock error or bias, $b$, is the amount that the receiver's clock is off. The receiver has four unknowns, the three components of GPS receiver position and the clock bias $[x, y, z, b]$. The equations of the sphere surfaces are given by:

$\left(\mathrm{x}-\mathrm{x}_{\mathrm{i}}\right)^{2}+\left(\mathrm{y}-\mathrm{y}_{\mathrm{i}}\right)^{2}+\left(\mathrm{z}-\mathrm{z}_{\mathrm{i}}\right)^{2}=\left[\left(\mathrm{t}_{\mathrm{r}}+\mathrm{b}-\mathrm{t}_{\mathrm{i}}\right) \mathrm{c}\right]^{2} \mathrm{i}=1,2,3, \ldots \ldots, \mathrm{n}$

or in terms of pseudo ranges, $\mathrm{p}_{\mathrm{i}}=\left(\mathrm{t}_{\mathrm{r}}+\mathrm{b}-\mathrm{t}_{\mathrm{i}}\right) \mathrm{c}$, as

$\mathrm{p}_{\mathrm{i}}=\sqrt{ }\left[\left(\mathrm{x}-\mathrm{x}_{\mathrm{i}}\right)^{2}+\left(\mathrm{y}-\mathrm{y}_{\mathrm{i}}\right)^{2}+\left(\mathrm{z}-\mathrm{z}_{\mathrm{i}}\right)^{2}\right]-\mathrm{bc}, \mathrm{i}=1,2,3, \ldots, \mathrm{n}$

These equations can be solved by algebraic or numerical methods.

\section{Benefits of an earthquake monitoring system}

An earthquake early-warning system may provide the critical information needed (1) to minimize loss of property and lives, (2) to aid rescue operations, and (3) to assist recovery from earthquake damage. The most effective use of earthquake early warning is to activate automated systems to prepare for incoming strong ground shaking. For example: slowing down rapid-transit vehicles and high-speed trains to avoid potential derailment, orderly shutdown of pipelines and gas lines to minimize fire hazards, controlled shutdown of manufacturing operations to decrease potential damage to equipment, and safe-guarding 6 computer information by saving vital information and retracting disk heads away from the disk surface. All the above can be accomplished to a useful extent with a few seconds notification. Although human response may take more than a few seconds, personal safety could be greatly enhanced if people were alerted: school children could seek cover under desks and workers could move away from hazardous positions. More importantly, early earthquake notification might reduce panic and confusion. The functions of a modern society, including civil and military operations, will be less likely to turn into chaos if an early earthquake notification is available and drills for appropriate actions have been performed. For example, the Mexico City Alert System (and associated programs to educate the public) demonstrated its usefulness during the September 14, 1995 earthquake (Espinosa-Aranda et al., 1995; 1996). For an earthquake early-warning system with sufficient numbers of well- distributed accelerometers (such as the real time system in operation in Taiwan), we can estimate quickly the maximum expected ground-motion caused by an earthquake (i.e., a shake map), so that emergency response teams may be dispatched where they are needed most. In practice, such a shake map will be revised and updated as more information is received. In addition, an inventory of man-made structures and their vulnerability must exist so that loss estimation from an earthquake can be quickly assessed to aid disaster response and recovery. The usefulness of this approach is recognized, especially after the Northridge earthquake (Goltz, 1996; Eguchi et al., 
1997). Recently, the Federal Emergency Management Agency (FEMA) of the United States introduced a risk assessment methodology -- Hazards United States (Hazus) to assist emergency managers in estimating earthquake risk in their jurisdictions (Nishenko, 1998). The shake map produced by an earthquake early warning or rapid notification system is required for Hazus' approach in estimating loss after an earthquake disaster. As noted before, few cities are favorable located so that an earthquake warning message can be effectively used before strong shaking occurs. Although Mexico City is favorably located for earthquake early warning, the experience of the SAS in Mexico City indicates that it is not easy to manage a public earthquake early warning system. Nevertheless, an earthquake early-warning or rapid notification system is an important tool to provide the necessary information (e.g., a shake map) for effective earthquake mitigation and to monitor the seismicity after a disastrous earthquake.

\section{Conclusion}

Despite the progress made in understanding the physics of the earthquake, the prediction of earthquake we can made today are inevitably uncertain mainly because of highly complex nature of the earthquake. But with the advancement through GPS system technology we can now mitigate the risk of disaster like earthquake.

Using GPS we could easily monitor the earthquake in real time and continuous frame. With this technology we could easily monitor the deformation on earth surface under the range $5 \mathrm{~mm}$ or les by monitoring the displacement curve with seismic released energy data and by monitoring the crustal stress.

\section{References}

[1] ISPRS Journal of Photogrammetry \& Remote Sensing. Volume 59, issue 4, June 2005, Pages 185-198. Remote Sensing \& Geospatial Information For Natural Hazard Characterization.

[2] Li HN, Huo LS. Recent developments of structural vibration control in civil engineering in China. Journal of Earthquake and Tsunami 2010; 4(1):9-21.

[3] Celebi M, Sanli A. GPS in pioneering dynamic monitoring of long-period structures. Earthquake Spectra 2002; 18(1):47-61.

[4] Kijewski-Correa T, Kochly M. Monitoring the wind-induced response of tall buildings: GPS performance and the issue of multipath effects. Journal of Wind Engineering and Industrial Aerodynamics 2007; 95 (9- 11):1176-1198.

[5] Chan WS, Xu YL, Ding XL, Xiong YL, Dai WJ. Assessment of dynamic measurement accuracy of GPS in three directions. ASCE Journal of Surveying Engineering 2006; 132(3):108-117.

[6] Roberts GW, Cosser E, Meng XL, Dodson A. High frequency deflection monitoring of bridges by GPS. Journal of Global Positioning Systems 2004; 3(1-2):226-231.

[7] Santerre R, Beutler G. A proposed GPS method with multi-antennae and single receiver. Journal of Geodesy 1993; 67(4):210-223.

[8] Ragheb AE, Edwards SJ, Clarke PJ. Using filtered and semi-continuous high rate GPS for monitoring deformations. Journal of Surveying Engineering 2010; 136(2):72-79.

[9] Sun Weijun and Li Gang: Research of GPS technology in seismic monitoring system.

[10] Kennedy, M. The Global Positioning System and GIS: An Introduction. Chelsea: MI, Ann Arbor Press, Inc., 1996.

[11] Fundamentals of GPS. Arlington: VA, Navtech Seminars, Inc.1996.

[12] Dana, P.H.: "Global Positioning System (GPS) Time Dissemination for Real-Time Applications", Kluwer Academic Publishers, 1997.

[13] Haggart, C.: "How GPS works: An Introduction".

[14] Dommety G. and Jain, R.: "Potential Networking Applications of Global Positioning Systems". 